\title{
A search for edge-on galaxy lenses in the CFHT Legacy Survey ${ }^{\star}$
}

\author{
J. F. Sygnet ${ }^{1,2}, \mathrm{H} . \mathrm{Tu}^{3,1,2}$, B. Fort ${ }^{1,2}$, and R. Gavazzi ${ }^{1,2}$ \\ 1 CNRS, UMR7095, Institut d'Astrophysique de Paris, 75014 Paris, France \\ e-mail: [sygnet; fort; gavazzi]@iap.fr \\ 2 UPMC Univ Paris 06, UMR7095, Institut d'Astrophysique de Paris, 75014 Paris, France \\ 3 Physics Department \& Shanghai Key Lab for Astrophysics, Shanghai Normal University, Shanghai 200234, PR China \\ e-mail: TuHong@shnu.edu.cn
}

Received 28 December 2009 / Accepted 18 April 2010

\section{ABSTRACT}

\begin{abstract}
Context. The new generation of wide-field optical imaging as the Canada France Hawaii Telescope Legacy Survey (CFHTLS) enables discoveries of all types of gravitational lenses present in the sky. The Strong Lensing Legacy Survey (SL2S) project has started an inventory of clusters or groups of galaxies lenses and of Einstein rings around distant massive ellipticals.

Aims. Here we attempt to extend this inventory by finding lensing events produced by massive edge-on disk galaxies that remain a poorly documented class of lenses.

Methods. We implemented and tested an automated search procedure of edge-on galaxy lenses in the CFHTLS Wide fields with magnitude $18<i<21$, inclination angle lower than $25^{\circ}$, and a photometric redshift determination. This procedure estimated the lensing convergence of each galaxy from the Tully-Fisher law and selected only those few candidates that exhibit a possibly nearby arc configuration at a radius compatible with this convergence $\left(r_{\text {arc }} \lesssim 2 r_{\mathrm{E}}\right)$. The efficiency of the procedure was tested after a visual examination of the whole initial sample of 30444 individual edge-on disks.

Results. After calculating the surface density of edge-on lenses possibly detected in a survey for a given seeing, we deduce that this theoretical number is about 10 for the CFHTLS Wide, a number in broad agreement with the 2 good candidates detected here. We show that the Tully-Fisher selection method is very efficient at finding valuable candidates, though its accuracy depends on the quality of the photometric redshift of the lenses. Finally, we argue that future surveys will detect at least a hundred of such lens candidates.
\end{abstract}

Key words. gravitational lensing: strong - dark matter - galaxies: spiral - galaxies: halos

\section{Introduction}

The $\Lambda$-CDM cosmological paradigm has been highly successful at explaining most of the large-scale properties of the Universe; nevertheless it still must be shown that this model is able to explain its small-scale properties. Among these are the formation and dark matter content of disk galaxies, and in particular the rotation curves of disks have played a key role as evidence that there is dark matter on small scales (e.g. Bosma 1978; Rubin et al. 1980; Persic et al. 1996; de Blok et al. 2008; Donato et al. 2009). Despite tremendous observational and modeling efforts over the past three decades, advances are still plagued by our ability to disentangle both the contributions of baryonic disk and bulges and the contribution of dark matter.

The hypothesis of the maximum bulge or disk (van Albada \& Sancisi 1986; Persic et al. 1996; Dutton et al. 2005) is able to fix the mass-to-light ratio of the stellar populations and therefore to put constraints on the dark matter content of galaxies or, alternatively, on inferences about modifications of the gravitation

^ Based on observations obtained with MegaPrime/MegaCam, a joint project of CFHT and CEA/DAPNIA, at the Canada-France-Hawaii Telescope (CFHT), which is operated by the National Research Council (NRC) of Canada, the Institut National des Sciences de l'Univers of the Centre National de la Recherche Scientifique (CNRS) of France, and the University of Hawaii. This work is based in part on data products produced at TERAPIX and the Canadian Astronomy Data Centre as part of the Canada-France-Hawaii Telescope Legacy Survey, a collaborative project of $\mathrm{NRC}$ and CNRS. law (e.g. de Blok \& McGaugh 1997; McGaugh 2004; de Blok et al. 2008; Donato et al. 2009). For further insight into the relative contribution of different species (stars in disk, stars in bulge, and dark matter), one needs additional constraints to break the degeneracies (e.g. Kranz et al. 2003; de Jong \& Bell 2007; Ibata et al. 2007).

Another particularly promising method resides in studying the dynamical properties of strong lensing galaxies. Mostly because of the scarcity of background QSOs, only very few lensing disk galaxies with a low enough redshift, suitable for kinematical observations, have been known until recently. For instance, one can mention the early studies of the Einstein Cross Q22370305 (Trott \& Webster 2002; Trott et al. 2010; van de Ven et al. 2008), of B1600+434 (Jaunsen \& Hjorth 1997; Koopmans et al. 1998; Maller et al. 2000), of J2004-1349 (Winn et al. 2003), of CXOCY J220132.8-320144 (Castander et al. 2006), or Q00453337 (Chieregato et al. 2007).

Great advances have become possible recently thanks to the advent of large high-quality imaging and spectroscopic datasets. Automated or visual inspection of high-resolution imaging from the Hubble Space Telescope (HST) (e.g. Marshall et al. 2009; Newton et al. 2009; Faure et al. 2008; Moustakas et al. 2007; Covone et al. 2009) or from the ground (e.g. Cabanac et al. 2007; Kubo \& Dell' Antonio 2008) have just started to provide us with many candidates for strong galaxy-galaxy lensing events. For spectroscopy, an automated search of superimposed spectra at different redshifts into a given fiber followed up by highresolution HST imaging turns out to be very efficient on large 
spectroscopic datasets like the Sloan Digital Sky Survey (SDSS) (Bolton et al. 2004, 2006, 2008; Auger et al. 2009; Willis et al. $2005,2006)$. These studies are limited to relatively low redshifts $(z \sim 0.2)$, and most of them focus on lensing by massive elliptical galaxies with a large splitting angle.

Conversely, late-type galaxies, while more numerous (e.g. Bartelmann 2000; Kochanek 2006; Chae 2010), have a smaller Einstein radius and are thus more difficult to identify. For instance the recently found edge-on disk galaxy OAC-GL J1223-1239 (Covone et al. 2009), with $r_{\mathrm{E}} \sim 0$.' 43, could only be found with HST imaging. A first automated spectroscopic search for disk galaxies lenses in the SDSS database was done by Féron et al. (2009): they found 8 candidates among 40000 disk galaxies, two of them being already confirmed as genuine lenses.

Despite these efforts, disk galaxies remain a poorly documented class of lenses, particularly for edge-on lensing galaxies. This situation calls for an improvement. We need more edge-on disk lenses with a low inclination to maximize the success of dynamical studies and simplify the recognition of lensing/lensed structures in imaging data. In this paper we investigate if the deep multiband sub-arcsecond imaging data of the Canada France Hawaii Telescope Legacy Survey (CFHTLS) offers a good opportunity to find edge-on disk lenses beyond the redshift range accessible by the SDSS.

The paper is organized as follows. Section 2 presents a calculation of the number of lenses with an $i$ magnitude $18<i<21$ and an inclination lower than $25^{\circ}$ which should be detected for a given seeing in an imaging survey. Section 3 explains the procedure that we have followed to extract edge-on lens candidates from the CFHTLS Wide survey by estimating an Einstein radius from the Tully-Fisher (TF) relation (Tully \& Fisher 1977) and comparing it with the arc radius possibly detected around the galaxy. We also cross-check the validity of this automated procedure by a visual inspection of all the edge-on galaxies present in the survey. In Sect. 4 we discuss our results with an overview on what could be done with future all-sky imaging surveys. Throughout this paper all magnitudes are expressed in the $\mathrm{AB}$ system, and we assume the concordance cosmological model with $h=0.7, \Omega_{\mathrm{m}}=0.3$, and $\Omega_{\Lambda}=0.7$.

\section{Forecasting the frequency of edge-on disk lenses}

We attempt here to predict the number of lenses that could be observed within an imaging survey of properties similar to the CFHTLS Wide.

\subsection{Cross-sections and optical depths}

We estimate lensing optical depths by starting from generic assumptions about mass distributions (see e.g. Turner et al. 1984; Schneider et al. 1992; Marshall et al. 2005; Kochanek 2006). In particular, we assume that all lenses are singular isothermal spheres (SIS) parameterized by their velocity dispersion $\sigma$. They are also assumed to be sparse enough that the probability of multiple lensing is negligible, so that one can sum up individual lensing cross-sections over the probed volume. Arguably, circular symmetry might not be a good description of edge-on disk galaxies. Bartelmann \& Loeb (1998) showed that, averaging over all possible inclination angles, the effect of the disk is null for the overall cross-section. It is however found that the efficiency of edge-on lenses is a bit boosted so that a calculation of the total SIS cross-section and the same calculation restricted to inclinations $\alpha_{i} \leq 25^{\circ}$ (corresponding to a third of all possible orientations) consistent with edge-on disks will bracket the right number. So we define a corrective term $1 / 3<\eta<1$ that will multiply the total lensing cross-section of disky galaxies approximated as SIS.

Ideally, the cross-section $X$ for multiple imaging by an SIS mass distribution at redshift $z_{1}$ of a source at $z_{\mathrm{s}}$ is given by the solid angle (in steradians) subtended by the Einstein radius:

$X=\pi r_{\mathrm{E}}^{2}=\pi\left[4 \pi\left(\frac{\sigma}{c}\right)^{2} \frac{D_{\mathrm{ls}}}{D_{\mathrm{os}}}\right]^{2}$

However, due to observational limitations, one cannot detect all the lenses of a given $r_{\mathrm{E}}$, the main limitation being seeing and blending with the light possibly coming from the foreground deflector. In addition, for an SIS profile, two images (arcs) are produced at radii $\theta_{1}$ and $\theta_{2}$ from the optical axis (with the convention $\left.\theta_{1}>\theta_{2}>0\right)$ so that the radius of the source is $\beta=\left(\theta_{1}-\theta_{2}\right) / 2$ and its Einstein radius is $r_{\mathrm{E}}=\left(\theta_{1}+\theta_{2}\right) / 2$. The detectability of a lens implies that the outermost arc $\theta_{1} \equiv r_{\text {arc }}>r_{\text {cut }}$, with $r_{\text {cut }}$ a limiting radius that we set to the seeing $F W H M r_{\text {cut }}=0{ }^{\prime} 8$. The other condition for multiple imaging, $\beta<r_{\mathrm{E}}$, complements the constraints for the actual lensing cross-section. The full calculation yields

$X^{\prime}=\left\{\begin{array}{lll}0 & \text { if } & r_{\mathrm{E}}<r_{\text {cut }} / 2 \\ \pi\left(2 r_{\mathrm{E}}-r_{\text {cut }}\right) r_{\text {cut }} & \text { if } & r_{\text {cut }} / 2 \leq r_{\mathrm{E}} \leq r_{\text {cut }} \\ \pi r_{\mathrm{E}}^{2} & \text { if } & r_{\text {cut }}<r_{\mathrm{E}} .\end{array}\right.$

The optical depth $\tau$ for strong lensing of a source at $z_{\mathrm{s}}$ can be written as the volume integral of the above cross-section integrated over the distribution of the velocity dispersion distribution function of lenses $\phi(\sigma)$ that is assumed to be constant with cosmic time (at least back to redshift $\sim 1$ beyond which lensing efficiency quickly falls off):

$\tau\left(z_{\mathrm{s}}\right)=\int_{0}^{z_{\mathrm{s}}} \mathrm{d} z_{1} f_{k}(\chi)^{2} \frac{\mathrm{d} \chi}{\mathrm{d} z_{1}} \int_{0}^{\infty} \mathrm{d} \sigma K\left(z_{\mathrm{l}}, \sigma\right) X^{\prime}\left(\sigma, z_{\mathrm{l}}, z_{\mathrm{s}}\right) \phi(\sigma)$.

In this equation we have introduced a selection function $K\left(z_{1}, \sigma\right)$ of potential lenses that will depend on observational settings and lens finding strategy. Notice that a purely source oriented approach, typical of radio surveys (Browne et al. 2003; Winn et al. 2003), will have $K\left(z_{1}, \sigma\right)=1$. Conversely a lens oriented survey like SLACS (Bolton et al. 2006) will be limited in redshift by the depth of SDSS spectroscopy and will pick the most massive $\sigma \sim 240 \mathrm{~km} \mathrm{~s}^{-1}$ galaxies from the start. Since we assume a flat cosmology we can use comoving distances $f_{k}(\chi)=\chi$ in Eq. (3). We use the velocity dispersion of Chae (2010) for SDSS late-type galaxies which reads

$\phi(\sigma)=\phi^{*} \sigma^{-1}\left(\frac{\sigma}{\sigma^{*}}\right)^{\alpha} \exp \left[-\left(\frac{\sigma}{\sigma^{*}}\right)^{\beta}\right] \frac{\beta}{\Gamma(\alpha / \beta)}$,

with $\alpha=0.69, \beta=2.10, \phi^{*}=0.066 h^{3} \mathrm{Mpc}^{-3}$ and $\sigma^{*}=$ $91.5 \mathrm{~km} \mathrm{~s}^{-1}$.

\subsection{Selection function of lenses}

As already anticipated, observational limitations will lead to a non-unity selection function $K\left(z_{1}, \sigma\right)$. Obviously we cannot directly select on $z_{1}$ or $\sigma$ but, more realistically, we use apparent magnitudes. Therefore we assume that a combination of photometric redshifts (with negligible errors at this stage) and a direct relation between luminosity and velocity dispersion will approximate most of the observational selections effects. More precisely, assuming that lens galaxies are isothermal spheres allows us to relate the velocity dispersion to the rotation velocity 
$\sigma=V_{\mathrm{c}} / \sqrt{2}$. We then use the latest normalization of the TullyFischer relation in the $R$ band from Pizagno et al. (2007) and neglect the intrinsic scatter about the mean relation, giving

$\log V_{\mathrm{c}}=-0.135\left(M_{r}-5 \log _{10} h+20.332\right)+2.210$.

In addition, we shift apparent $i$ band magnitudes back to the rest frame $r$ band using a redshift dependent k-correction so that $M_{r}=i-25-5 \log _{10}\left(D_{\text {lum }} / h^{-1} \mathrm{Mpc}\right)-k_{i \rightarrow r}$. A polynomial fit to the photometric change $k_{i \rightarrow r}$ yields

$k_{i \rightarrow r}(z)=-0.36+0.65 z-0.92 z^{2}+z^{3}+0.06 z^{4}$,

which is obtained by redshifting template of Sbc galaxies from Coleman et al. (1980) using hyperz facility (Bolzonella et al. 2000). Hence a given selection in magnitude $i_{\min }<i<i_{\max }$ can readily be cast into a redshift-dependent selection in velocity dispersion: $K\left(z_{1}, \sigma\right)=\Theta\left(\sigma-\sigma_{\min }\left(z_{1}\right)\right) \Theta\left(\sigma_{\max }\left(z_{1}\right)-\sigma\right)$, where $\Theta$ is the Heavyside step function.

\subsection{Population of background sources}

Given the lensing optical depth of Eq. (3) and a redshift distribution of sources, the number of lenses can be written as

$N=\eta A n_{0} \int_{0}^{\infty} \mathrm{d} z_{\mathrm{s}} p\left(z_{\mathrm{s}}\right) \tau\left(z_{\mathrm{s}}\right)$,

where $A$ is the surveyed area and $\eta$ the term accounting for the boost of the disk introduced in Sect. 2.1. In principle the redshift distribution inferred from photometric redshifts by Coupon et al. (2009) for the CFHTLS Wide survey should be most suitable for our analysis. However, magnification bias, which can create a gain of more than one magnitude in depth, makes better suited the redshift distribution of the HST COSMOS survey down to $i<26$, which is closer to the population of faint blue objects we aim at detecting behind foreground edge-on disks. Therefore we use the results of Leauthaud et al. (2007) from COSMOS that can be approximated by the expression:

$\frac{\mathrm{d} n\left(z_{\mathrm{s}}\right)}{\mathrm{d} z_{\mathrm{s}}}=\frac{1}{z_{0} \Gamma(a)} \mathrm{e}^{-z_{\mathrm{s}} / z_{0}}\left(z_{\mathrm{s}} / z_{0}\right)^{a-1}$,

with $z_{0}=0.345$ and $a=3.89$ (Gavazzi et al. 2007). In addition, at these limiting $i<26$ magnitudes, one can achieve a surface density of sources of about $n_{0}=300000 \mathrm{deg}^{-2}$.

\subsection{Results}

For the values considered above, we plot in Fig. 1 the predicted number of edge-on disks as a function of the cut radius which is identified to the seeing. For the CFHTLS Wide image quality (seeing $\simeq 0$.' 8 in $g$ band) and depth we typically get about 0.07-0.30 detectable lenses per square degree depending on the edge-on disk boost factor $\eta$. Therefore for the $124 \mathrm{deg}^{2}$ imaged in five $u^{*}$ griz bands, one might discover between 8 and 37 lenses assuming all our hypotheses (SIS density profile, no scatter in the TF relation nor errors with photometric redshifts...) are correct.

To assess our calculations we also made predictions for the number of lensing elliptical galaxies assuming a perfect relation between magnitude and velocity dispersion as given by the Faber-Jackson relation whose normalization is taken from Oguri (2006). Down to a limiting AB magnitude $i<22.5$ for the deflector, a similar calculation based on the velocity dispersion function for ellipticals also given by Chae (2010) yields the red curve

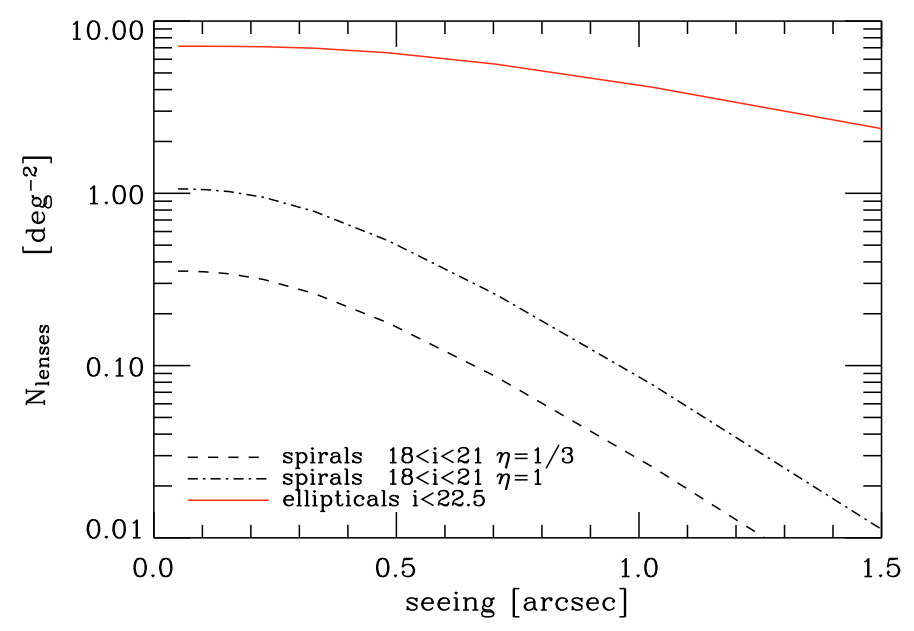

Fig. 1. Predicted number of gravitational lenses per square degree as a function of image quality. Imaging survey specifications in terms of depth are those of the CFHTLS Wide. The dashed and dot-dashed black lines bracket the number of lensing edge-on disks depending on the edge-on disk boost factor $\eta$. The upper solid red curve is for lensing by bright $i<22.5$ ellipticals.

in Fig. 1. It predicts 10 to 100 more lenses with a substantially lower dependence on seeing below 1'.5. This might be explained by the generally larger Einstein radius (due to greater velocity dispersions) of ellipticals for which the exponential fall-off of the distribution functions drops beyond 1'.5. Worse seeing will also make the detectability of arcs and counterimages much more challenging beyond $0 .{ }^{\prime} 8$. In addition, the light from the deflector, no matter the seeing size, will prevent many small-scale detections. For comparison, Marshall et al. (2005) predicted about 50 lenses that could be detected by SNAP (two magnitudes deeper

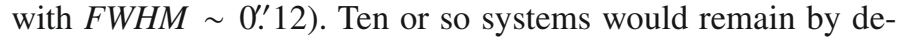
grading depth and image quality to our survey specifications and by restricting ourselves to bright $i<22.5$ deflectors. This is consistent with our calculations. This is also in broad agreement with COSMOS observations of strong lensing luminous $M_{r}<-20$ ellipticals (Faure et al. 2008).

\section{Seeking edge-on disk lenses within the CFHTLS Wide}

From the calculations above, we can undertake a comprehensive search of lenses in the CFHTLS Wide survey. The T0005 data release (for a detailed description, see Mellier et al. 2008) spans 171 fields of one square degree each for which images in at least the three $g, r$, and $i$ bands are obtained. But, in order to calculate the photometric $z_{1}$ of the possible lenses, we restrict ourselves to the 124 fields for which the five $u^{*}, g, r$, $i$, and $z$ bands are present. The typical $80 \%$ completeness limit magnitude for point sources is 25.4, 25.4, 24.6, 24.5, and 23.6 respectively. Image quality is also very good with typical seeing FWHM of 0.'90, 0.'85, 0.'75, 0.'72, and 0.71 respectively.

\subsection{Photometric preselection of bright edge-on galaxies}

We start from a catalog of 28 million extended objects detected with SExtractor (Bertin \& Arnouts 1996). We first select 1.02 million objects within the magnitude range $18<i<21$ as the initial data base. The bright $i<18$ low redshift galaxies are excluded because their angular size is too large as compared to their expected Einstein radius. Objects fainter than $i=21$ are 
also discarded because of the low signal-to-noise and the difficulty of future spectroscopic follow-up. The few already known spiral galaxies producing multiply-imaged QSOs happened to be in this magnitude range (Koopmans et al. 1998; Maller et al. 2000; Castander et al. 2006; Chieregato et al. 2007).

Edge-on disk galaxies are then preselected as highly elongated objects with projected ellipticity $0.7<e \equiv\left(a^{2}-b^{2}\right) /\left(a^{2}+\right.$ $\left.b^{2}\right)<0.92$. The lower limit on the ellipticity is chosen because it removes most of the ellipticals (Park et al. 2007) while the upper limit $e<0.92$ stands for rejecting most ( $\sim 90 \%)$ of spurious objects like diffraction patterns of bright stars. This ellipticity cut yields 30444 bright edge-on galaxies.

\subsection{The Tully-Fischer relation as a proxy for Einstein radius}

The multiband ( $u^{*}$ griz) photometry of the survey allows the computation of photometric redshifts (Coupon et al. 2009). Along with apparent magnitudes, this additional information enables estimates of absolute magnitudes that can readily be converted into an estimate of their rotation velocity $V_{\mathrm{c}}$ using the latest calibrations of the Tully-Fischer relation (Pizagno et al. 2007). Assuming the lensing galaxy can be approximated by a circularly symmetric isothermal mass distribution, the velocity dispersion is $\sigma=V_{\mathrm{c}} / \sqrt{2}$. Using a typical source redshift $1<z_{\mathrm{s}}<3$ and taking into account a possible error $\Delta M_{r}=-0.37$ on the absolute magnitude (corresponding to the $1 \sigma$-dispersion of the TF law), we can predict an Einstein radius $r_{\mathrm{E}(\mathrm{TF})}$ that is then compared to the seeing size. More specifically, given that the maximum distance of the furthest of the two multiple images of a source is twice its Einstein radius and assuming that this latter image could not be detected if embedded within the seeing disk, we have selected foreground objects by requiring they satisfy the TF cut: $2 r_{\mathrm{E}(\mathrm{TF})} \geq 00^{\prime} 8$. Applying this last automated cut on the catalog provides us with 2064 massive edge-on disk galaxies with the greatest chance of being lenses.

\subsection{Subsequent visual classification}

At this stage, we were unable to avoid visual inspection of color cutout images of the above 2064 objects. The first reason for that is that the small size of Einstein radii and therefore distance of multiple images to the central deflector is comparable to both the deflector size and seeing disk. Hence SExtractor will fail at deblending arcs and deflector. Consequently we undertook a systematic inspection of these 2064 objects and decided to further consider objects that fulfill the following criteria:

(i) Arcs between 0.' 6 and 3.'5: The inner limit is set by the separability of arc and deflector as said above whereas the outer limit is thought to be just above the innermost radius accessible to the automated arc detectors used by the SL2S collaboration for detecting large separation group and cluster-scale lenses (Cabanac et al. 2007). Note that we discard arcs that are definitely too dim for spectroscopic follow-up (estimated to correspond to a magnitude $i \gtrsim 24$ ).

(ii) Arc and deflector colors are different: In order to reduce the contamination by faint satellite galaxies falling into the main galaxy and producing tidal tails, we require the color of the arc and the deflector to be different. This criterium will not be $100 \%$ efficient at removing close pairs but its impact on completeness is very low because the probability that a lens and a source at very different redshifts have the same color is almost negligible. Since precise photometry is
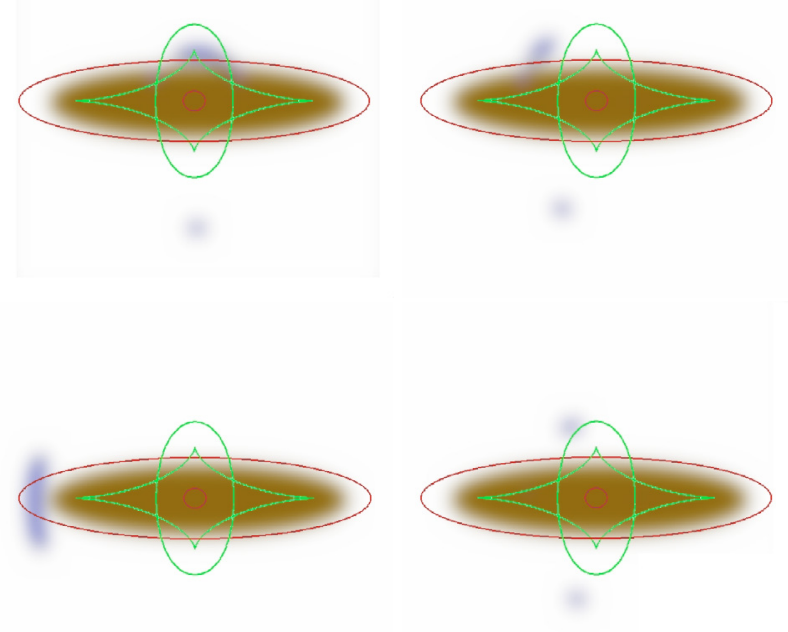

Fig. 2. Families of multiple images configurations for lensing by an edge-on disk (yellow/dark) galaxy for different source (blue/light) positions. Red/dark lines (resp. green/light) represent critical lines (resp. caustics) in the image (resp. source) plane. From top left to bottom right: "Bulge arcs", "Fold arcs", "Disk arcs" and "Pairs" (Shin \& Evans 2007).

difficult on such small scales, color estimation is essentially based on visual inspection of the color images.

(iii) Should look like a library of lensed features: Finally, the most stringent criterium that we apply is based on the geometry of the arc and its counterimages, as almost all ${ }^{1}$ edge-on disk lenses produce very typical image configurations (see e.g. Keeton \& Kochanek 1998; Bartelmann \& Loeb 1998; Shin \& Evans 2007) that we can classify into four families "Bulge arcs", "Fold arcs", "Disk arcs" and "Pairs" as suggested by Shin \& Evans (2007) and illustrated in Fig. 2. Naturally, intermediate cases between these categories may exist with about the same probability for an edge-on disk galaxy similar to the Milky Way (Shin \& Evans 2007).

At this stage we obtain 11 candidates labeled with a A or B upper script in Table 1 and also shown in the upper panels panels of Fig. 4.

(iv) We then further require that the arc radius is smaller than twice the Einstein radius estimated with the Tully-Fisher method as discussed in Sect. 3.2.

This latter filter (hereafter the TF (iv) test) leaves us with only 2 galaxies labeled A in Table 1 which would be our prime candidates for follow-up observations: they occupy the first row of Fig. 4. We notice that, no matter whether we consider class A or $\mathrm{A}+\mathrm{B}$ candidates, we find numbers that are roughly consistent with our calculation of Sect. 2 although we fully acknowledge that completeness cannot be achieved given the sharp cutoffs we used.

1 The observation of faint counterimages close to the center is very challenging, especially for disk galaxies with substantial dust extinction (Kochanek 2006). 

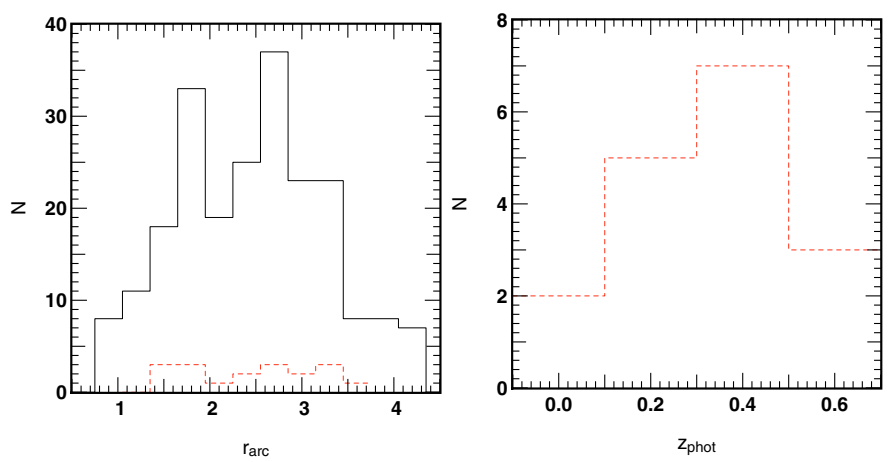

Fig. 3. Left panel: distribution of arc radii for the 190 peculiar lines of sight (solid black curve) of Sect. 3.4, and for the 16 best candidates (dashed red curve) listed in Table 1. Right panel: photometric redshift distribution for these 16 candidates.

\subsection{Manual cross-check of the automated procedure}

In order to validate the above procedure and to assess the effects of photometric redshift uncertainties, we decided to cross-check it by visually analyzing the complete sample of 30444 objects of the CFHTLS selected at the end of Sect. 3.1. The number of objects is large but a trained observer, working in sessions of about two hours a day ${ }^{2}$, is able to explore about 1000 subimages per hour, corresponding to $4 \mathrm{deg}^{2}$ per hour. This procedure would be impossible for an all-sky survey but it translate into about $30 \mathrm{~h}$ for the whole CFHTLS Wide and allows an interesting cross-check of the automated procedure. We obviously apply the same criteria described above in Sect. 3.3. For information, the first of these steps: (i) An edge-on galaxy must have a faint object nearby, allows us to get rid of more than $99.3 \%$ of the potential lenses. It leaves us with 190 interesting galaxies (i.e. $1.5 \pm 0.5$ per $\mathrm{deg}^{2}$ ) that deserve closer inspection, using steps (ii) and (iii).

The full visual selection procedure allowed us to keep only 16 candidates. Of them, 11 were already selected as class A or $\mathrm{B}$ in the more automated procedure above whereas 5 additional systems were found to be worth presenting here although they don't pass the Tully-Fisher tests (neither the first cut $2 r_{\mathrm{E}(\mathrm{TF})} \geq 0$.' 8 nor the second TF (iv) test $r_{\mathrm{arc}} \leq 2 r_{\mathrm{E}(\mathrm{TF})}$ ). These class $C$ objects occupy the bottom of Table 1 and Fig. 4, while Fig. 3 shows the photometric redshift distribution and the distribution of arc radii for the full 16 candidates. This timeconsuming cross-check therefore validates our use of the TF tests, and shows that errors on photometric redshifts are not, in our case, a major source of concern.

\section{Results and discussion}

\subsection{Description of the candidates}

We have seen that our search of edge-on disk galaxies in $124 \mathrm{deg}^{2}$ of the CFHTLS Wide yields 2 A-class good candidates (listed at the top of Table 1 and Fig. 4). We believe they are the only genuine strong lenses detected by our procedure:

- SL2SJ021711-042542 is a spiral galaxy with a photometric redshift $z_{1}=0.57 \pm 0.06$. It is a bulge arc configuration with an observed arc radius $\left(r_{\text {arc }}=11^{\prime \prime}\right.$. 7$)$ fully compatible with the maximum arc radius allowed by a TF model $\left(2 r_{\mathrm{E}(\mathrm{TF})}=1{ }^{\prime \prime} .8\right)$.

- SL2SJ022533-042414 is also a spiral with a photometric redshift $z_{1}=0.24 \pm 0.04$. It is a bulge arc configuration at

\footnotetext{
2 This was found to be a decent limit to preserve human eye accuracy. Repeated visual inspections of several fields were done at different periods to assess the stability of the human decision process.
}

$r_{\text {arc }} \simeq 1$ 1.'4 with a faint red counter image which is compatible with the maximum arc radius of the TF model $\left(2 r_{\mathrm{E}(\mathrm{TF})}=\right.$ 1.'4).

From a purely visual selection (i.e. not applying filter (iv)), we would have selected 9 more candidates (labeled B in Table 1). These uncertain candidates can be roughly classified in two categories:

- $85 \%$ of them have an arc radius that is too large for their lens magnitude and a small color difference with their lens. They probably correspond to stellar tidal trails produced by galaxy interactions or disrupted satellites. For the most massive satellites falling in the centre of a galaxy potential these structures can be bright enough to be observed, especially when star formation is initiated by gravitational interactions. These thin structures are wrapped around the dark matter halo potential and can mimic arc structures. Some of them are seen straddling the edge-on disk like an arc triplet formed by a naked cusp. We see in Fig. 3's left panel a cut-off for $r_{\text {arc }}>2$ 2.5. Its existence shows that these frequent spurious detections are more easily rejected for large arc radii. Only the high spatial resolution of HST images would discard them at smaller radius.

- The remaining 15\% (namely SL2SJ020933-054012 and SL2SJ140454+544552) correspond probably to Singly Highly Magnified Sources (SHMS) where the arc is formed at a distance greater than $2 r_{\mathrm{E}}$ and does not produce multiple images. The optical depth of this strong flexion regime was studied by Keeton et al. (2005) with both isothermal and NFW halo density profiles. He found that the proportion of SHMS among arcs should be less than $20 \%$ for distant background sources. For these candidates we shall remember that the baryonic mass within the disk can increase the convergence of the edge-on lens beyond the one considered for the TF test (Bartelmann \& Loeb 1998). Therefore if the arc is not too far from the critical line an observer shall not systematically discard them without further considerations.

We also listed 5 class $\mathrm{C}$ candidates at the bottom of Table 1 which have been selected only during the cross-check manual procedure (Sect. 3.4), because their theoretical Tully-Fisher radius $r_{\mathrm{E}(\mathrm{TF})}$ is less than half the seeing value. We believe they are interesting systems anyway and are worth presenting here.

\subsection{Discussion and prospects}

During this detection exercise, several lessons were found, which can be of some use for future surveys. The Tully-Fisher cut turns out to be an efficient way to strongly decrease the number of line of sight to be visually scrutinized and to get rid of many of the false positive cases. Indeed no bona fide strong lens was found during the manual cross-check (Sect. 3.4) out of the 30 444-2064 objects discarded by the TF cut (Sect. 3.2). However we stress that the accuracy of this cut is limited by the quality of photometric redshifts. With a limited number of colors, high redshifts could be mistaken for low ones, specially in the range $0.05<z_{1}<0.15$ (e.g. Bernstein \& Huterer 2009), therefore the absolute magnitude of the lens would be over estimated and thus resulting in too small a value of $r_{\mathrm{E}(\mathrm{TF})}$. We therefore recommend not to implement a too stringent TF cut.

We show in the left panel of Fig. 3 the distribution of arc radii. The effect of the seeing cut-off at small radii can be clearly seen: the numerous lenses with arc radius smaller than the seeing disk $\left(0{ }^{\prime} 8\right)$ are missed. The photometric redshift distribution of the 16 selected candidates lies in the range $0.2 \lesssim z_{1} \lesssim 0.6$ as 


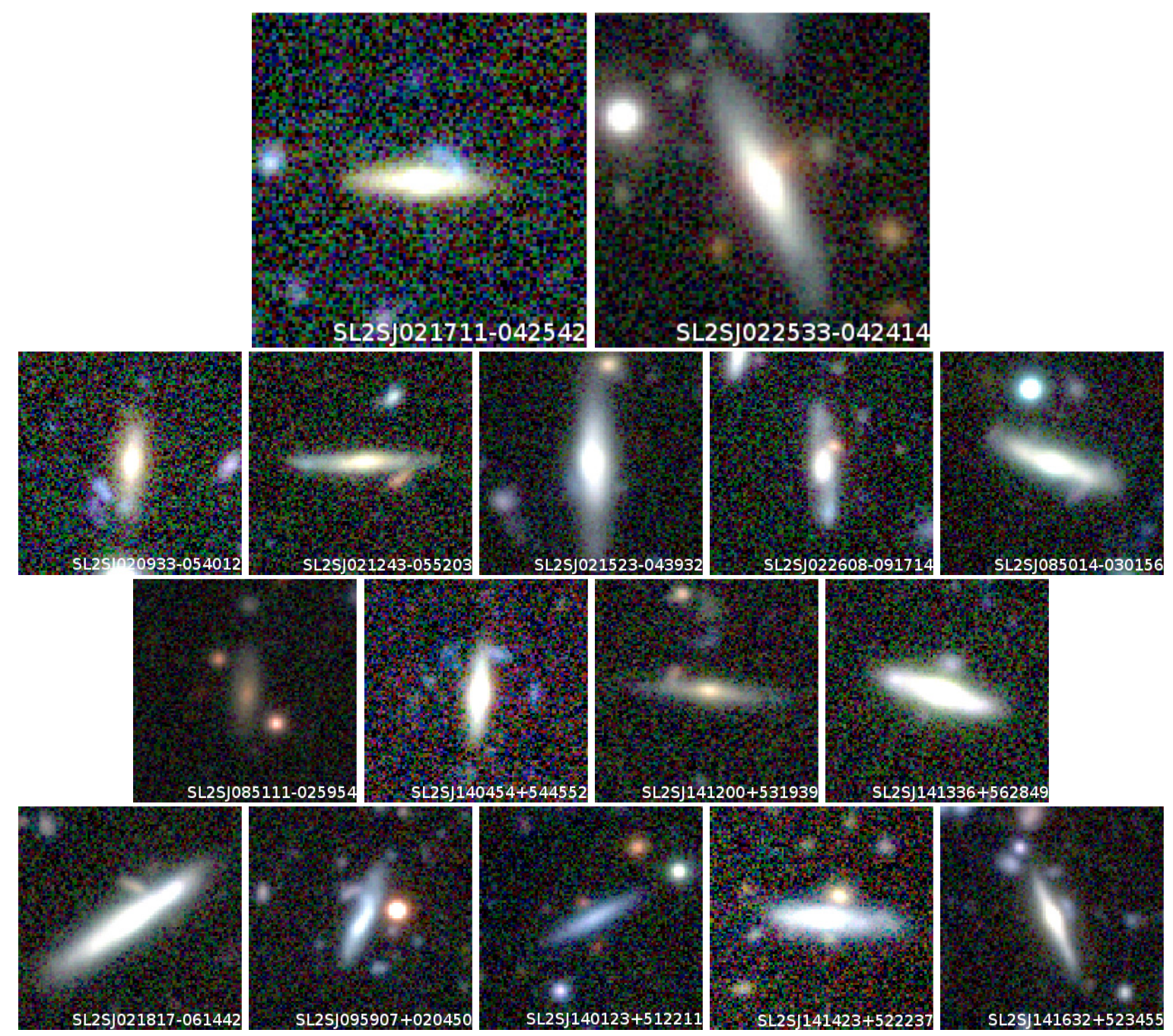

Fig. 4. Color images of the 16 candidates. Image sizes are all 18.' 6 on a side. First row: class A objects passing the Tully-Fischer (iv) test. Second and third rows: good B quality systems that do not pass the TF (iv) test. Fourth row: additional class $\mathrm{C}$ systems found during manual cross-check.

Table 1. Edge-on disk lens candidates found in the CFHTLS Wide survey.

\begin{tabular}{|c|c|c|c|c|c|c|c|c|c|c|c|}
\hline Name & $\begin{array}{l}\text { RA (J2000) } \\
\text { (hr min sec) }\end{array}$ & $\begin{array}{c}\operatorname{Dec}(\mathrm{J} 2000) \\
\left({ }^{\circ},{ }^{\prime \prime}\right)\end{array}$ & $u^{*}$ & $g$ & $\begin{array}{c}A B \text { mag } \\
r\end{array}$ & $i$ & $z$ & $\begin{array}{l}r_{\text {arc }} \\
\left({ }^{\prime \prime}\right)\end{array}$ & $z_{1}$ & Family & $\begin{array}{c}r_{\mathrm{E}(\mathrm{TF})} \\
\left(^{\prime \prime}\right)\end{array}$ \\
\hline SL2SJ021711-042542 ${ }^{A}$ & $02: 17: 11.843$ & $-04: 25: 42.00$ & 22.80 & 21.60 & 20.43 & 19.72 & 19.35 & 1.7 & $0.57 \pm 0.06$ & $b$ & 0.90 \\
\hline SL2SJ022533-042414 ${ }^{A}$ & $02: 25: 33.129$ & $-04: 24: 14.99$ & 20.79 & 19.50 & 18.68 & 18.18 & 17.85 & 1.4 & $0.24 \pm 0.04$ & $b / d$ & 0.70 \\
\hline SL2SJ020933-054012 & $02: 09: 33.633$ & $-05: 40: 12.99$ & 23.12 & 21.75 & 20.39 & 19.75 & 19.42 & 3.3 & $0.44 \pm 0.04$ & $f$ & 0.61 \\
\hline SL2SJ021243-055203 ${ }^{B}$ & $02: 12: 43.801$ & $-05: 52: 03.23$ & 22.68 & 21.38 & 20.29 & 19.76 & 19.44 & 3.0 & $0.35 \pm 0.06$ & $f / d$ & 0.44 \\
\hline SL2SJ021523-043932 & $02: 15: 23.801$ & $-04: 39: 32.33$ & 21.14 & 19.64 & 18.76 & 18.18 & 17.89 & 2.2 & $0.21 \pm 0.04$ & $b / f$ & 0.58 \\
\hline SL2SJ022608-091714 ${ }^{B}$ & $02: 26: 08.138$ & $-09: 17: 14.87$ & 22.35 & 21.16 & 20.11 & 19.52 & 19.11 & 1.8 & $0.46 \pm 0.06$ & $f$ & 0.76 \\
\hline SL2SJ085014-030156 ${ }^{B}$ & $08: 50: 14.572$ & $-03: 01: 56.08$ & 21.73 & 20.35 & 19.21 & 18.57 & 18.17 & 3.1 & $0.44 \pm 0.04$ & $d$ & 1.28 \\
\hline SL2SJ085111-025954 ${ }^{B}$ & $08: 51: 11.743$ & $-02: 59: 54.21$ & 23.79 & 22.71 & 21.49 & 20.74 & 20.23 & 3.3 & $0.55 \pm 0.04$ & $p$ & 0.44 \\
\hline SL2SJ140454+544552 $2^{B}$ & $14: 04: 54.217$ & $+54: 45: 52.49$ & 22.79 & 21.17 & 19.92 & 19.30 & 18.86 & 3.6 & $0.41 \pm 0.04$ & $d$ & 0.74 \\
\hline SL2SJ141200+531939 ${ }^{B}$ & $14: 12: 00.934$ & $+53: 19: 39.83$ & 22.38 & 21.33 & 20.35 & 19.81 & 19.55 & 3.2 & $0.42 \pm 0.06$ & $d$ & 0.55 \\
\hline SL2SJ141336+562849 ${ }^{B}$ & $14: 13: 36.001$ & $+56: 28: 49.25$ & 21.39 & 20.01 & 19.11 & 18.60 & 18.26 & 2.3 & $0.27 \pm 0.04$ & $b$ & 0.63 \\
\hline SL2SJ021817-061442C & $02: 18: 17.050$ & $-06: 14: 42.59$ & 20.13 & 18.93 & 18.44 & 18.04 & 17.91 & 2.8 & $0.07 \pm 0.06$ & $d$ & 0.13 \\
\hline SL2SJ095907+020450 & 09:59:07.769 & $+02: 04: 50.30$ & 22.22 & 21.46 & 21.25 & 20.97 & 20.84 & 2.8 & $0.07 \pm 0.06$ & $f$ & 0.22 \\
\hline SL2SJ140123+512211 ${ }^{C}$ & $14: 01: 23.392$ & $+51: 22: 11.00$ & 22.24 & 21.22 & 20.86 & 20.47 & 20.31 & 1.4 & $0.14 \pm 0.10$ & $p$ & 0.08 \\
\hline SL2SJ141423+522237 ${ }^{C}$ & $14: 14: 23.150$ & $+52: 22: 37.46$ & 21.02 & 19.94 & 19.48 & 19.27 & 19.08 & 1.9 & $0.17 \pm 0.04$ & $p$ & 0.22 \\
\hline SL2SJ141632+523455 ${ }^{C}$ & $14: 16: 32.336$ & $+52: 34: 55.14$ & 22.86 & 21.62 & 20.78 & 20.35 & 19.96 & 1.6 & $0.30 \pm 0.06$ & $b$ & 0.25 \\
\hline
\end{tabular}

Notes. The uncertainty on magnitudes is, in almost every case, on the order of 0.01 and the Einstein radius is the maximum value allowed by the TF law. For geometry family, $b$ stands for "bulge arc", $f$ for "fold arc", $d$ for "disk arc" and $p$ for "pair" (see Sect. 3.3).

${ }^{(A)}$ Candidates found with both the automated and the manual cross-check procedures and satisfying the Tully-Fisher (iv) test. ${ }^{(B)}$ Candidates same as ${ }^{(A)}$, but failing the Tully-Fisher (iv) test. ${ }^{(C)}$ Extra candidates found only with the manual cross-check procedure. 
shown in the right panel of Fig. 3. In this respect, our CFHTLS candidates nicely complement the ones extracted from shallower surveys like SDSS (see e.g. Féron et al. 2009).

We can wonder if the LSST (LSST Collaboration 2009) survey will significantly improve the situation. It will cover 20000 square degrees of the sky and will reach the depth of the CFHTLS Wide just after the first year of observation and the depth of the CFHTLS Deep after ten years (Ivezić et al. 2008). The seeing quality at Cerro Pachon in Chile is expected to be 0.7 quite comparable to the one at CFHT. Therefore, in theory, we can expect from our calculation (cf. Fig. 1) at least 1300 massive edge-on lenses in LSST. Its deeper photometry will also allow to get better photometric redshift and hence to improve the chance of keeping good candidates with the TF test. Nevertheless, conservatively assuming the same detection probability as in CFHTLS, one expects to find in the LSST about 1770 candidates instead of the $11 \mathrm{~A}+\mathrm{B}$ class candidates found here, leading probably to about 320 bona-fide edge-on lenses, which is an excellent perspective.

The situation shall improve even more dramatically with future space surveys like JDEM or Euclid (Marshall et al. 2005; Refregier 2009) which will have a better resolution $(<0$. 3). We expect from our calculation that these surveys could detect a few thousand edge-on lenses with Einstein radius substantially smaller than in ground based surveys.

\section{Conclusion}

In conclusion, while it is extremely difficult to find edge-on lenses with ground based imaging surveys, the situation is not completely hopeless with sub-arcsecond seeing $(<0$.' 8$)$. A good way to implement a fast search procedure is to preselect a limited subset of galaxies presumably massive enough to produce a detectable arc. The Tully-Fisher law seems quite appropriate for this purpose, since it can predict an Einstein radius for each disk. Galaxies with the largest Einstein radii are then checked for the presence of a faint source nearby which might be a gravitational arc. Extensive visual cross-checking of all disk galaxies present in the survey has shown that this procedure picks most of the interesting candidates, provided reliable photometric redshifts are available.

Whatever the final choice of an observer, the number of candidates that can be choosen for an observational follow up is quite small: here we ended up with only 2 good candidates from 124 square degrees of the CFHTLS Wide. This illustrates how challenging it is to dig out such lenses from any ground based survey. Despite their great number in the sky and as long as wide space surveys are not available, edge-on disk lenses will remain scarce and precious objects for astronomers who try to understand the relative distribution of dark and ordinary matter in spirals.

Acknowledgements. The authors are thankful to the CFHTLS members and the Terapix team for their excellent work in reducing and distributing data to the community. They would also like to thank P. Marshall and J.-P. Kneib for fruitful discussions, and J. Coupon for providing his photometric redshifts calculations. Part of this work was supported by the Agence Nationale de la Recherche (ANR) and the Centre National des Etudes Spatiales (CNES). Part of this project is done under the support of the National Natural Science Foundation of China Nos. 10878003, 10778752, Shanghai Foundation No. 07dz22020, and the Leading Academic Discipline Project of Shanghai Normal University (08DZL805).

\section{References}

Auger, M. W., Treu, T., Bolton, A. S., et al. 2009, ApJ, 705, 1099

Bartelmann, M. 2000, A\&A, 357, 51
Bartelmann, M., \& Loeb, A. 1998, ApJ, 503, 48

Bernstein, G., \& Huterer, D. 2009, MNRAS, 1648

Bertin, E., \& Arnouts, S. 1996, A\&AS, 117, 393

Bolton, A. S., Burles, S., Schlegel, D. J., Eisenstein, D. J., \& Brinkmann, J. 2004, AJ, 127, 1860

Bolton, A. S., Burles, S., Koopmans, L. V. E., Treu, T., \& Moustakas, L. A. 2006, ApJ, 638, 703

Bolton, A. S., Burles, S., Koopmans, L. V. E., et al. 2008, ApJ, 682, 964

Bolzonella, M., Miralles, J.-M., \& Pelló, R. 2000, A\&A, 363, 476

Bosma, A. 1978, Ph.D. Thesis, Groningen Univ.

Browne, I. W., Wilkinson, P. N., Jackson, N. J. F., et al. 2003, MNRAS, 341, 13

Cabanac, R. A., Alard, C., Dantel-Fort, M., et al. 2007, A\&A, 461, 813

Castander, F. J., Treister, E., Maza, J., \& Gawiser, E. 2006, ApJ, 652, 955

Chae, K. 2010, MNRAS, 402, 2031

Chieregato, M., Miranda, M., \& Jetzer, P. 2007, A\&A, 474, 777

Coleman, G. D., Wu, C., \& Weedman, D. W. 1980, ApJS, 43, 393

Coupon, J., Ilbert, O., Kilbinger, M., et al. 2009, A\&A, 500, 981

Covone, G., Paolillo, M., Napolitano, N. R., et al. 2009, ApJ, 691, 531

de Blok, W. J. G., \& McGaugh, S. S. 1997, MNRAS, 290, 533

de Blok, W. J. G., Walter, F., Brinks, E., et al. 2008, AJ, 136, 2648

de Jong, R. S., \& Bell, E. F. 2007, in Island Universes - Structure and Evolution of Disk Galaxies, ed. R. S. de Jong, 107

Donato, F., Gentile, G., Salucci, P., et al. 2009, MNRAS, 397, 1169

Dutton, A. A., Courteau, S., de Jong, R., \& Carignan, C. 2005, ApJ, 619, 218

Faure, C., Kneib, J.-P., Covone, G., et al. 2008, ApJS, 176, 19

Féron, C., Hjorth, J., McKean, J. P., \& Samsing, J. 2009, ApJ, 696, 1319

Gavazzi, G., Fumagalli, M., Cucciati, O., Boselli, A. 2010, A\&A, accepted [arXiV: 1003.3795$]$

Gavazzi, R., Treu, T., Rhodes, J. D., et al. 2007, ApJ, 667, 176

Ibata, R., Martin, N. F., Irwin, M., et al. 2007, ApJ, 671, 1591

Ivezić, Ž., Axelrod, T., Becker, A. C., et al. 2008, in AIP Conf. Ser. 1082, ed. C. A. L. Bailer-Jones, 359

Jaunsen, A. O., \& Hjorth, J. 1997, A\&A, 317, L39

Keeton, C. R., \& Kochanek, C. S. 1998, ApJ, 495, 157

Keeton, C. R., Kuhlen, M., \& Haiman, Z. 2005, ApJ, 621, 559

Kochanek, C. S. 2006, in Saas-Fee Advanced Course 33: Gravitational Lensing: Strong, Weak and Micro, ed. G. Meylan, P. Jetzer, P. North, P. Schneider, C. S. Kochanek, \& J. Wambsganss, 91

Koopmans, L. V. E., de Bruyn, A. G., \& Jackson, N. 1998, MNRAS, 295, 534

Kranz, T., Slyz, A., \& Rix, H. 2003, ApJ, 586, 143

Kubo, J. M., \& Dell'Antonio, I. P. 2008, MNRAS, 385, 918

Leauthaud, A., Massey, R., Kneib, J.-P., et al. 2007, ApJS, 172, 219

LSST Collaboration 2009, unpublished, [arXiv: 0912.0201]

Maller, A. H., Simard, L., Guhathakurta, P., et al. 2000, ApJ, 533, 194

Marshall, P., Blandford, R., \& Sako, M. 2005, New Astron. Rev., 49, 387

Marshall, P. J., Hogg, D. W., Moustakas, L. A., et al. 2009, ApJ, 694, 924

McGaugh, S. S. 2004, ApJ, 609, 652

Mellier, Y., Bertin, E., Hudelot, P., et al. 2008, The CFHTLS T0005 Release,

http://terapix.iap.fr/cplt/oldSite/Descart/ CFHTLS-TQ005-Release.pdf

Moustakas, L. A., Marshall, P., Newman, J. A., et al. 2007, ApJ, 660, L31

Newton, E. R., Marshall, P. J., \& Treu, T. 2009, ApJ, 696, 1125

Oguri, M. 2006, MNRAS, 367, 1241

Park, C., Choi, Y.-Y., Vogeley, M. S., Gott, J. R. I., \& Blanton, M. R. 2007, ApJ, 658, 898

Persic, M., Salucci, P., \& Stel, F. 1996, MNRAS, 281, 27

Pizagno, J., Prada, F., Weinberg, D. H., et al. 2007, AJ, 134, 945

Refregier, A. 2009, Experimental Astronomy, 23, 17

Rubin, V. C., Ford, W. K. J., \& Thonnard, N. 1980, ApJ, 238, 471

Schneider, P., Ehlers, J., \& Falco, E. E. 1992, Gravitational Lenses (Berlin: Springer-Verlag)

Shin, E. M., \& Evans, N. W. 2007, MNRAS, 374, 1427

Trott, C. M., \& Webster, R. L. 2002, MNRAS, 334, 621

Trott, C. M., Treu, T., Koopmans, L. V., \& Webster, R. L. 2010, MNRAS, 401, 1540

Tully, R. B., \& Fisher, J. R. 1977, A\&A, 54, 661

Turner, E. L., Ostriker, J. P., \& Gott, III, J. R. 1984, ApJ, 284, 1

van Albada, T. S., \& Sancisi, R. 1986, Royal Soc. London Philos. Trans. Ser. A, 320,447

van de Ven, G., Falcon-Barroso, J., McDermid, R. M., et al. 2008, [arXiv:0807.4175]

Willis, J. P., Hewett, P. C., \& Warren, S. J. 2005, MNRAS, 363, 1369

Willis, J. P., Hewett, P. C., Warren, S. J., Dye, S., \& Maddox, N. 2006, MNRAS, 369,1521

Winn, J. N., Hall, P. B., \& Schechter, P. L. 2003, ApJ, 597, 672 